\title{
Efectos de la administración temprana de ácido tranexámico en la mortalidad, histerectomías y otras morbilidades en mujeres con hemorragia posparto: un ensayo clínico internacional, aleatorio, doble ciego y controlado contra placebo. Una revisión crítica
}

Effect of early tranexamic acid administration on mortality, hysterectomy, and other morbidities in women with post-partum haemorrhage (WOMAN): an international, randomised, double-blind, placebo-controlled trial. A critical review

Diego Nualart ${ }^{1,2}$, Martín Friedman ${ }^{1,2}$, Héctor J. Lacassie ${ }^{1,3}$

\begin{abstract}
Introduction: Post-partum haemorrhage remains as the first cause of maternal mortality worldwide. Tranexamic acid can reduce bleeding during surgery by up to a third of cases. Objectives: To assess the impact of tranexamic acid administration on maternal death and hysterectomy due to post partum haemorrhage, in addition to other relevant outcomes. Methods: Double blind placebocontrolled clinical trial of 20,060 women 16 years of age and older, diagnosed with postpartum haemorrhage. This was defined as bleeding over $500 \mathrm{ml}$ for vaginal deliveries or over $1,000 \mathrm{ml}$ in the case of cesarean section, or bleeding that significantly affected hemodynamic parameters. A first dose of $1 \mathrm{~g}$ of tranexamic acid or placebo was given at randomization; if bleeding persisted after $30 \mathrm{mi}-$ nutes or restarted within the first 24 hours, a second dose was given. Subgroup
\end{abstract}

\section{Key words:}

Postpartum hemorrhage, tranexamic acid, maternal mortality, histerectomy, anesthesia
División de Anestesiología Escuela de Medicina, Facultad

de Medicina, Pontificia Universidad Católica de Chile. Residente de Anestesiología.

Profesor Asociado en Anestesiología.

Fecha de recepción: 10 de octubre de 2018

Fecha de aceptación: 15 de octubre de 2018

Conflicto de intereses: Los autores declaran no tener conflicto de intereses.
Fuente de financiamiento: Los autores no recibieron patrocinio para llevar a cabo este artículo.

ORCID

https://orcid.org/0000-0001-5758-4113

Correspondencia:

Hector J. Lacassie

Marcoleta 377, $4^{\circ}$ piso, Departamento de Anestesiología Hospital Clínico UC, Santiago, Chile.

Teléfono: +56-2-2354-3270

Email: lacassie@med.puc.cl 
analysis wes performed for patients randomized within three hours of diagnosis. Results: Administration of tranexamic acid within the first three hours decreased maternal death due to bleeding by one third. Administration afterwards did not impact on mortality. No decrease in the risk of hysterectomy was found, but fewer laparotomies for control of postpartum haemorrhage were performed. There were no significant differences in embolic events or other adverse events between groups. Conclusion: The use of tranexamic acid in the first three hours of postpartum hemorrhage decreases maternal death by one third and should be included in the first-line management of postpartum haemorrhage.

\section{RESUMEN}

Introducción: La hemorragia posparto sigue siendo la primera causa de muerte materna a nivel mundial. El ácido tranexámico disminuye en cerca de un tercio el sangrado durante el acto quirúrgico. Objetivos: Evaluar el efecto de la administración de ácido tranexámico en la muerte materna e histerectomía por hemorragia posparto, además de otros resultados relevantes. Métodos: Estudio clínico controlado contra placebo. Se asignaron 20.060 mujeres mayores de 16 años con diagnóstico de hemorragia posparto (sangrado sobre $500 \mathrm{ml}$ en el caso de parto o sobre $1.000 \mathrm{ml}$ en caso de cesárea) o que afectara la hemodinamia. Recibieron $1 \mathrm{~g}$ de ácido tranexámico o placebo. Si el sangrado persistía después de $30 \mathrm{~min}$ o reiniciaba dentro de las primeras $24 \mathrm{~h}$ recibían una segunda dosis. Se realizó un análisis de subgrupo del beneficio de la intervención en pacientes que la recibían antes o después de 3 horas. Resultados: La administración de ácido tranexámico dentro de las primeras tres horas disminuyó en un tercio las muertes maternas por hemorragia. No hubo diferencias al administrar después de tres horas. No hubo disminución del riesgo de histerectomía, pero sí de laparotomías para el control de hemorragias posparto. No hubo diferencias significativas de eventos embólicos ni otros eventos adversos entre grupos. Conclusión: El uso del ácido tranexámico en las primeras tres horas de la hemorragia posparto disminuye en un tercio las muertes maternas y debiera incluirse en el manejo de primera línea de hemorragia posparto.

\section{Palabras clave:}

Ácido tranexámico, histerectomia, mortalidad materna, hemorragia posparto

\section{Introducción}

- ste trabajo presenta un análisis crítico del artículo de WOMAN Trial Collaborators[1].

La hemorragia obstétrica es la primera causa de muerte materna en el mundo. El daño tisular durante el parto puede promover la liberación del activador de plasminógeno tisular, el que convierte el plasminógeno en la enzima fibrinolítica plasmina. El uso de ácido tranexámico inhibe esta activación y su uso ha demostrado ser exitoso en el contexto de trauma y de otras cirugías de gran recambio de volumen.

\section{Objetivo del estudio}

Evaluar el efecto de la administración de ácido tranexámico en la muerte materna e histerectomía por hemorragia posparto, además, de otros resultados relevantes.

\section{Diseño del estudio}

Se realizó un estudio clínico aleatorio, doble ciego, controlado contra placebo, llevado a cabo entre marzo de 2010 y abril de 2016, en 193 hospitales de 21 países de África, Asia, Europa y Sudamérica.

Se incluyeron 20.060 mujeres mayores de 16 años con diagnóstico clínico de hemorragia posparto, definida como sangrado estimado mayor a $500 \mathrm{ml}$ en el parto vaginal y mayor a $1.000 \mathrm{ml}$ en la cesárea o de una cuantía suficiente para afectar la estabilidad hemodinámica. Los criterios de inclusión fueron si el equipo tratante estimaba que la paciente debía recibir ácido tranexámico como parte del manejo en el contexto de una hemorragia posparto y que se encontraba recibiendo todos los cuidados necesarios para el 
manejo del evento.

Las pacientes fueron asignadas aleatoriamente a recibir $1 \mathrm{~g}$ de ácido tranexámico endovenoso o placebo. En caso de persistir la hemorragia 30 min después o recidivar dentro de las primeras $24 \mathrm{~h}$, recibieron una segunda dosis, igual a la primera.

Desenlace primario: Un agregado de muerte materna por cualquier causa o histerectomía dentro de 42 días desde la aleatorización.

Desenlaces secundarios: El principal desenlace secundario fue la muerte materna por hemorragia posparto. Otros desenlaces importantes incluyeron: eventos trombóticos (trombosis venosa profunda, tromboembolismo pulmonar, infarto al miocardio y accidentes cerebrovasculares); intervenciones quirúrgicas (tamponamiento intrauterino, embolización, suturas hemostáticas, ligadura arterial, histerectomía y laparotomía para control de la hemorragia); otras complicaciones (sepsis, falla renal, falla cardíaca, etc.) y eventos adversos, entre otros. Se hizo, además, un análisis de subgrupo en el grupo de hemorragia respecto de aquellas pacientes que recibieron la droga antes de las tres horas de iniciado el cuadro y aquellas que lo recibieron después.

\section{Resultados}

El ácido tranexámico disminuyó significativamente el riesgo de muerte materna por sangrado (155 [1,5\%] de 10.036 pacientes vs 191 [1,9\%] de 9.985 en el grupo placebo, (riesgo relativo [RR] 0,81, IC $95 \% 0,65-0,99 ; p=0,045)$, reduciendo substancialmente este riesgo en aquellas pacientes que lo recibían dentro de las primeras 3 horas desde el parto (89 [1,2\%] de ácido tranexámico vs 127 [1,7\%] del grupo placebo, RR 0,69, IC 95\% 0,52-0,91; p=0,008). La reducción del riesgo relativo RRR de mortalidad materna por sangrado con el uso de ácido tranexámico es de $19,3 \%$, con una reducción absoluta del riesgo de $0,4 \%$. El número necesario para tratar (NNT) para este desenlace es de 270 (IC 95\% 135-19.182). No se detectó diferencia en el riesgo de histerectomía con el uso de ácido tranexámico (RR 1,02, IC 95\% 0,88$1,07 \mathrm{p}=0,84$ ), aunque sí hubo una disminución de las laparotomías realizadas para el control del sangrado (RR 0,64, IC 95\% 0,49-0,85 p=0,002). No hubo diferencias en los eventos adversos, incluyendo los de naturaleza tromboembólica.

\section{Comentarios de los revisores}

Dado que en nuestro país al igual que a nivel mundial la muerte por hemorragia postparto sigue siendo una causa significativa de mortalidad, cualquier esfuerzo por su disminución debe dirigir nuestra atención, sobre todo con estas intervenciones que muestran un bajo riesgo para el paciente y un bajo importe económico.

Es un estudio de buena calidad metodológica, con descripción apropiada de los criterios de inclusión y exclusión, prospectivo, aleatorizado, doble ciego, con grupos a tratar comparables, con seguimiento completo y análisis por intención a tratar. El registro de los resultados fueron obtenidos de todas las pacientes aleaotorizadas (ácido tranexámico vs control) desde su ficha clínica. El tamaño muestral fue apropiadamente calculado y se hizo un análisis de subgrupo de acuerdo al tipo de parto (vaginal vs cesárea) y al tiempo en que se realizó la intervención (antes o después de $3 \mathrm{~h}$ ).

Las poblaciones estudiadas incluyeron países de alto y bajo nivel de ingreso, diversos fenotipos y áreas variadas del mundo, incluyendo Sudamérica. El medicamento usado se encuentra ampliamente disponible en nuestro medio y el mundo, lo que le da una buena validez externa a sus resultados.

\section{Conclusión y recomendación}

Este es un estudio con bajo riesgo de sesgo, se respeta la aleatorización de las pacientes incluidas, tiene una baja pérdida de participantes durante el estudio, con buen control de las variables. Además, cuenta con un excelente número y variedad de pacientes, que concuerda con la evidencia de otros estudios relacionados[2] y con plausibilidad fisiopatológica. Nos parece que existe evidencia suficiente para realizar un cambio en la práctica clínica habitual y recomendar el uso de este medicamento como tratamiento de primera línea en la hemorragia posparto, tanto en parto vaginal como cesárea. Si bien el NNT y su IC 95\% es amplio, hay que tener en cuenta que el costo de la intervención es bajo, sin efectos secundarios descritos lo que nos lleva a plantear su uso por el eventual beneficio que proporciona a nuestros pacientes en la reducción de mortalidad por hemorragia. Este cambio en conducta podría evitar hasta un tercio de las muertes maternas sin agregar morbilidad ni imponer una carga económica mayor. Hasta el momento no existen estudios similares con otros agentes antifibrinolíticos que nos permitan comparar la eficacia o versus el ácido tranexámico. 


\section{Referencias}

1. Shakur H, Roberts I, Fawole B, Chaudhri R, El-Sheikh M, Akintan $A$, et al. Effect of early tranexamic acid administration on mortality, hysterectomy, and other morbidi- ties in women with post-partum haemorrhage (WOMAN): an international, randomised, double-blind, placebo-controlled trial. Lancet. 2017:1-12.

2. Shakur H, Roberts I, Bautista R, Caballero J, Coats T, Dewan Y, et al. Effects of tranexamic acid on death, vascular occlusive events, and blood transfusion in trauma patients with significant haemorrhage (CRASH-2): a randomised, placebo-controlled trial. Lancet. 2010;376(9734):23-32. 